\title{
LIST OF MANUSCRIPTS \\ AND DICTATIONS
}

Preliminary Manuscripts and Dictations, 1870-1905

1870 [The Tennessee Land] $\sigma_{I}$

1877 [Early Years in Florida, Missouri] 64

1885 The Grant Dictations 66

The Chicago G.A.R. Festival 67

[A Call with W.D. Howells on General Grant] 70

Grant and the Chinese 72

Gerhardt 74

About General Grant's Memoirs 75

[The Rev. Dr. Newman] 99

1890, 1893-94 The Machine Episode IOI

1897 Travel-Scraps I 107

1898 Four Sketches about Vienna II8

[Beauties of the German Language] II 8

[Comment on Tautology and Grammar] III

[A Group of Servants] $\quad$ I20

[A Viennese Procession] $\quad$ I24

1898 My Debut as a Literary Person 127

1898-99 Horace Greeley 145

1898-99 Lecture-Times 146

1898-99 Ralph Keeler I5o

1900 Scraps from My Autobiography. From Chapter IX ISS

1900 Scraps from My Autobiography. Private History of a Manuscript That Came to Grief ${ }^{16} 64$

1903 [Reflections on a Letter and a Book] I8I

1903 [Something about Doctors] ${ }_{1} 88$

1904 [Henry H. Rogers] 192

1905 [Anecdote of Jean] Ig9 


\section{AUTOBIOGRAPHY OF MARK TWAIN}

\begin{tabular}{|c|c|c|c|}
\hline 1906 & \multicolumn{3}{|c|}{ An Early Attempt 203} \\
\hline $1897-98$ & \multicolumn{3}{|c|}{ My Autobiography [Random Extracts from It] 203} \\
\hline 1906 & \multicolumn{3}{|c|}{ The Latest Attempt 220} \\
\hline 1906 & \multicolumn{3}{|c|}{ The Final (and Right) Plan 220} \\
\hline 1906 & \multicolumn{3}{|c|}{ Preface. As from the Grave $22 I$} \\
\hline \multirow[t]{5}{*}{1904} & \multicolumn{3}{|c|}{ The Florentine Dictations 222} \\
\hline & \multicolumn{3}{|l|}{ [John Hay] 222} \\
\hline & \multicolumn{3}{|c|}{ Notes on "Innocents Abroad" 225} \\
\hline & \multicolumn{3}{|c|}{ [Robert Louis Stevenson and Thomas Bailey Aldrich] } \\
\hline & \multicolumn{3}{|c|}{ [Villa di Quarto] 230} \\
\hline \multirow[t]{17}{*}{1906} & \multicolumn{3}{|c|}{ Autobiographical Dictations, January-March 250} \\
\hline & 9 January 250 & 7 February 337 & 8 March 396 \\
\hline & 10 January 254 & 8 February $34 I$ & 9 March 400 \\
\hline & 11 January 260 & 9 February 346 & 12 March 403 \\
\hline & 12 January 267 & 12 February 350 & 14 March 407 \\
\hline & 13 January 273 & 13 February 354 & 15 March 409 \\
\hline & 15 January 276 & 14 February 357 & 16 March 417 \\
\hline & 16 January 283 & 15 February 359 & 20 March $42 I$ \\
\hline & 17 January 287 & 16 February 363 & 21 March 429 \\
\hline & 18 January 292 & 20 February 367 & 22 March 432 \\
\hline & 19 January 294 & 21 February 369 & 23 March 436 \\
\hline & 23 January 302 & 22 February 373 & 26 March 439 \\
\hline & 24 January 315 & 23 February 376 & 27 March 446 \\
\hline & 1 February 319 & 26 February 379 & 28 March $45 I$ \\
\hline & 2 February 323 & 5 March 385 & 29 March 455 \\
\hline & 5 February 328 & 6 March 389 & 30 March 462 \\
\hline & 6 February 334 & 7 March 392 & \\
\hline
\end{tabular}


This page intentionally left blank 